Herz 2013 · 38:567-568

DOI 10.1007/s00059-013-3928-6

Online publiziert: 15. August 2013

๑) Urban \& Vogel 2013

\title{
U.C. Hoppe
}

Department of Internal Medicine II, Paracelsus Medical University Salzburg, Salzburg

\section{Extrakardiale Einflüsse auf das Herz und die Herzinsuffizienz}

zwischen Herz und Niere. L. Großekettler und Kollegen stellen Pathomechanismen, die zum prä- bzw. intrarenalen Nierenversagen bei Herzinsuffizienz führen können, und differenzialtherapeutische Möglichkeiten beim kardiorenalen Syndrom dar. Nach Ausschöpfen einer sequenziellen Nephronblockade muss bei therapierefraktärer Hypervolämie, auch wenn keine dialysepflichtige Niereninsuffizienz besteht, eine Nierenersatztherapie erwogen werden. Bei schwerer Herzinsuffizienz mit chronisch progredienter Niereninsuffizienz scheint die Peritonealdialyse hämodynamisch im Vergleich zu extrakorporalen Verfahren vorteilhaft. Randomisierte Studien liegen jedoch nicht vor.

Für die Belastungsintoleranz bei Herzinsuffizienz ist eine Vielzahl pathophysiologischer Mechanismen verantwortlich. Den aktuellen Stand zum Einfluss von körperlichem Training auf die zentrale Hämodynamik, vaskuläre Veränderungen, die neurohumorale Aktivierung und den Skelettmuskelmetabolismus fassen F. Edelmann und B. Pieske zusammen. Zudem diskutieren die Kollegen geeignete Trainingsmodalitäten für Patienten mit Herzinsuffizienz. Anders als einer Metaanalyse kleiner Studien zufolge konnte in der randomisierten HF-ACTION-Studie durch körperliches Training bei HFrEF keine Senkung der Sterblichkeit erzielt werden; es fand sich aber eine Besserung der Belastungstoleranz. Bei stabiler Herzinsuffizienz wird körperliches Training als Teil eines umfassenden Behandlungskonzepts in nationalen und internationalen Leitlinien empfohlen.
Patienten mit einer Herzinsuffizienz und zusätzlich Depression, Anämie oder Schlafapnoe-Syndrom haben eine schlechtere Prognose als Patienten ohne diese Komorbiditäten. Die Kolleginnen J. Wallenborn und C. Angermann, die federführend die MOOD-HF-Studie durchführen, fassen die Datenlage zu dem wichtigen Thema der komorbiden Depression bei Herzinsuffizienz zusammen. Das Screening zur Detektion einer Depression ist wenig aufwendig. Derzeit fehlt jedoch die Evidenz, dass eine antidepressive Therapie über eine Steigerung des seelischen Wohlbefindens hinaus auch die Morbidität oder Mortalität beeinflusst. Antworten hierauf soll die laufende MOOD-HF-Studie liefern.

Bei herzinsuffizienten Patienten ist eine Anämie meist multifaktoriell bedingt. S. Reda und Kollegen legen in ihrem Beitrag dar, dass häufig eine supprimierte Eisenverfügbarkeit und -aufnahme, eine gestörte Erythropoetinfreisetzung und -wirkung wie auch eine Hämodilutionsanämie bestehen. Eine intravenöse Eisensubstitution wird in Leitlinien bei symptomatischen Patienten mit HFrEF und einem Eisendefizit entsprechend der FAIR-HFStudie empfohlen. Eine Therapie mit Stimulanzien der Erythropoietinproduktion ist hingegen nach derzeitiger Datenlage nicht indiziert.

Patienten mit Schlafapnoe-Syndrom entwickeln gehäuft eine Herzinsuffizienz; andererseits begünstigt eine Herzinsuffizienz das Auftreten eines obstruktiven oder zentralen Schlafapnoe-Syndroms, wie T. Plenge und J. Müller-Ehmsen in ihrem Beitrag darstellen. Eine CPAP-Be- 
handlung kann das obstruktive Schlafapnoe-Syndrom bessern, ist bei der zentralen Form jedoch nicht ausreichend. Als Alternative wird bei Herzinsuffizienz mit Schlafapnoe-Syndrom in Studien die adaptive Servoventilation, aber auch eine elektrische Stimulation des Nervus phrenicus zur Zwerchfellkontraktion getestet.

Der klinische Verlauf von Patienten mit Herzinsuffizienz ist zudem vom Körpergewicht abhängig. Während eine Adipositas als kardiovaskulärer Risikofaktor gilt, leben bei einer Herzinsuffizienz übergewichtige Patienten länger als solche mit niedrigem Gewicht, was auch als „Adipositas-Paradox" bekannt ist. Dabei scheint ein hoher relativer Anteil fettfreier Masse entscheidend zu sein. Eine Kachexie, an deren Genese unter anderem inflammatorische Prozesse beteiligt sind, ist hingegen wie bei anderen chronischen Erkrankungen mit einem ungünstigen Verlauf assoziiert. Die Kollegen Clauser und Altenberger geben einen aktuellen Überblick über die Bedeutung des Körpergewichts bei Patienten mit Herzinsuffizienz. Erste Substanzen, die gezielt die Muskelmasse bei Herzinsuffizienz erhalten bzw. aufbauen sollen, wurden bisher in Tiermodellen und kleinen Patientengruppen untersucht.

Dieses Heft von Herz soll dazu beitragen, die Bedeutung von Komorbiditäten für Patienten mit Herzinsuffizienz weiter in das klinische Bewusstsein zu bringen. Laufende Studien werden hoffentlich kausale Therapieoptionen in diesem Bereich eröffnen.

Uta C. Hoppe

\section{Korrespondenzadresse}

\section{Prof. Dr. U.C. Hoppe}

Department of Internal Medicine II, Paracelsus Medical University Salzburg Müllner Hauptstr. 48, A-5020 Salzburg Österreich

u.hoppe@salk.at 